\title{
On exponential consensus for time-varying non-cooperative nonlinear networks
}

\author{
S. Manfredi* and D. Angeli ${ }^{\diamond}$
}

\begin{abstract}
In this paper we present new results on asymptotic consensus for continuous-time nonlinear time varying networks. A key feature in the following is that the monotonicity property (i.e. cooperativity) is not required, unlike most of existing literature on the subject. Moreover, we give an estimate of the exponential rate of convergence towards the agreement manifold.
\end{abstract}

keyword: nonlinear networks, Consensus, Multi agent systems.

\section{INTRODUCTION}

In recent years the scientific community has devoted considerable attention to the consensus problem (see [2], [1], [10], [3] [4] and references therein). The main feature of the above solutions is to allow every agent to autonomously converge to a common agreement value or trajectory about some variables of interest using only local information at the node and/or the information received from neighboring agents. In the literature different conditions have been proposed to assess consensus in discrete and continuous time ([6], [12], [13], [14], [5], [9], just to cite a few) and under different class of both nonlinear time invariant and switching/time varying networks as outlined in [18], [11], [21], [22], [19], [20]. Most of the above frameworks assumed (in implicit or explicit way) the property of cooperativity (that in turn implies monotonicity). Given a function $f(t, x)$ : $\mathbb{R} \times \mathbb{R}^{n} \rightarrow \mathbb{R}^{n}$, piecewise continuous in $t$ and locally Lipschitz continuous with respect to $x$, the associated system of differential equations $\dot{x}(t)=f(t, x(t))$, is called cooperative if for any $i \in\{1,2, \ldots, n\}, f_{i}(t, x)$ is non-decreasing with respect to $x_{j}$ for all $j \neq i$. Notice that this condition implies monotonicity of the flow $\phi\left(t ; t_{0}, x_{0}\right)$ with respect to initial conditions, namely, for all $t_{0}$ and all $t \geq t_{0}$, it holds $\phi\left(t ; t_{0}, x_{1}\right) \geq \phi\left(t ; t_{0}, x_{2}\right)$ if $x_{1} \geq x_{2}$ (where " $\geq "$ is meant componentwise), [24]. This property is usually guaranteed by requiring the sign definiteness of off diagonal entries of the Jacobian matrix

*S. Manfredi is with the Department of Electrical and Information Technology, University of Naples Federico II, Italy and with the Control and Power Group, Electrical and Electronic Engineering Department, Imperial College, London. Email: sabato.manfredi@unina.it

${ }^{\diamond} \mathrm{D}$. Angeli is with the Control and Power Group, Electrical and Electronic Engineering Department, Imperial College, London and with the Dip. di Sistemi e Informatica, University of Florence, Italy. Email: d.angeli@imperial.ac.uk

This work was supported in part by mobility program of University of Naples Federico II
$F(x)$ (i.e. [22]). An extension to the case of signed graph with both positive and negative entries of the Jacobian matrix is reported in [23], although the Jacobian has to fulfil the sign constant (i.e. $F_{i j}\left(x_{1}\right) F_{i j}\left(x_{2}\right) \geq 0, \forall$ $x_{1} x_{2} \in \mathbb{R}^{n}$ and $i, j \in N$ ) and sign-symmetric (i.e. $\left.F_{i j}(x) F_{j i}(x) \geq 0 \forall x \in \mathbb{R}^{n}\right)$ assumptions. Additionally it is still required the system to be monotone ([24]) with respect to the partial order induced by some arbitrary orthant.

\section{A. Paper Contribution}

In a recent paper [16], we introduced a condition for asymptotic agreement (state frozen integral connectivity), suitable for nonlinear time varying monotone networks that extended to this scenario the notion of integral connectivity introduced by Moreau for linear networks [15] with the additional merit to be frozen in state variables and therefore of simpler verification. Herein we undertake a non trivial further step that greatly extends the class of considered systems by removing the cooperativity assumption (and therefore the system flow monotonicity). Specifically the paper contributions are: i) guaranteed exponential consensus under weak connectivity properties (just existence of a spanning tree for a suitable averaged graph is required) for a large class of nonlinear time-varying non cooperative networks. This encompasses most of the agents models normally adopted in the literature in the linear and non linear time varying setting. Moreover, both the dynamic at the node (self-feedback) and the coupling can be time varying and state dependent with the notable feature that the strength of attraction between two agents may vanish as so as the distance between the state values becomes larger. This is representative of many interactions occurring in several network scenarios: for instance in social networks, agents with different opinions have a low mutual influence when their distance increases; in sensor networks the data coming from nodes at higher distance may be less weighted in data fusion operations at the node; ii) we extend the use of a "State Frozen" concept [16] and integral connectivity to this non-trivial scenario of non cooperative networks by introducing a suitable agents connectivity property (later called "Frozen integral connectivity"). This has the merit to avoid the circular argument by which solutions depend 
on the connectivity and the latter is in turn influenced by state evolutions. This type of circular argument normally makes up for conditions that can hardly be tested in the case of time-varying nonlinear agent dynamics and coupling without explicit apriori knowledge of solutions; iii) for the described class of systems, we provide an estimate of the exponential rate of convergence towards the agreement manifold.

\section{NOTATION AND PROBLEM STATEMENT}

Throughout the paper all vectors are assumed to be column vectors. To denote vectors we write $x=$ $\left[x_{1}, \ldots, x_{n}\right]$ for the column vector $x \in \mathbb{R}^{n} .|x|$ denotes the Euclidean norm of $x . \mathbf{1}$ is the vector of all ones and $e_{j}$ is the $j$-th element of the canonical basis of $\mathbb{R}^{n}$, where $n$ should normally be clear from the context. The integer interval $N=\{1,2, \ldots, n\}$ will be identified with the set of interacting agents.

Consider a network of agents as described by the following system of nonlinear differential equations:

$$
\dot{x}(t)=f(t, x(t))
$$

where $x(t) \in \mathbb{R}^{n}$ is the state vector, $t \in \mathbb{R}_{+}$denotes time and $f$ is a vector field $f: \mathbb{R}_{+} \times \mathbb{R}^{n} \rightarrow \mathbb{R}^{n}$ describing the dynamics of the interaction between agents.

We assume: i) $f$ is locally Lipschitz continuous with respect to $x$ uniformly in time, viz. for all compacts $K \in$ $\mathbb{R}^{n}$ there exists $L_{K}>0$, such that, for all $x_{a}, x_{b} \in K$ and all $t \geq 0$ it holds $\left|f\left(t, x_{a}\right)-f\left(t, x_{b}\right)\right| \leq L_{K} \mid x_{a}-$ $x_{b} \mid ;^{1}$ ii) that $f$ admits an agreement equilibrium set, that is:

$$
\mathcal{E}=\operatorname{span}\{\mathbf{1}\} \subseteq\left\{x \in \mathbb{R}^{n}: f(t, x)=0 \forall t \in \mathbb{R}_{+}\right\} .
$$

The assumptions on $f$, imply the local existence and the unicity of the system's solution on some maximally extended open interval of definition.

Let $x(t)$ denote a solution of (1). At any time instant $t$ the following quantities are of interest:

$$
x_{\max }(t)=\max _{k \in N}\left\{x_{k}(t)\right\}, \quad x_{\min }(t)=\min _{k \in N}\left\{x_{k}(t)\right\}
$$

and $\delta_{k}(t)=\left|x_{\max }(t)-x_{k}(t)\right|$ for all $k \in N$ (or symmetrically $\left.\tilde{\delta}_{k}(t)=\left|x_{\min }(t)-x_{k}(t)\right|\right)$.

Fixed an arbitrary solution $x(\cdot)$ and an arbitrary time $t$ we define a time-dependent permutation $p_{j}(t)$ of indeces $j \in N$ such that it fulfills

$$
x_{p_{1}(t)}(t) \leq x_{p_{2}(t)}(t) \leq x_{p_{3}(t)}(t) \leq \ldots \leq x_{p_{n}(t)}(t) .
$$

Notice that, if two or more entries of $x$ take some given value, then the permutation is not uniquely defined. Nevertheless the permutation always exists and the value $x_{p_{i}(t)}$ is independent of how it is selected. Therefore, for

\footnotetext{
${ }^{1}$ This holds, for instance, when the Jacobian is uniformly bounded as a function of time.
}

any solution $x(t)$ of (1) we can define the corresponding re-ordered solution as $x_{p_{i}(t)}$. At any time instant $t$ the following quantities are of interest:

$$
x_{\max }(t)=\max _{k \in N}\left\{x_{k}(t)\right\} ; \quad x_{\min }(t)=\min _{k \in N}\left\{x_{k}(t)\right\} .
$$

\section{Main Results}

Next we state our connectivity assumption which is crucial to attain asymptotic consensus.

\section{Assumption 1 (Frozen Integral Connectivity) Given} network (1), we say that Integral frozen connectivity holds, provided for all compact intervals $\mathcal{K} \subset \mathbb{R}$ there exist some sufficiently large $T>0$, positive $\varepsilon_{T}, \varepsilon_{\mathcal{K}}$, a root node $r \in N$, a rooted spanning tree $\mathcal{T}_{r} \subset N \times N$ and associated connectivity sets $\psi_{(i, j)} \subset \mathbb{R}$ (defined for $\left.(i, j) \in \mathcal{T}_{r}\right)$ such that for all $x \in \mathcal{K}^{n}$ and any $t \geq 0$

$$
\begin{aligned}
& \operatorname{sign}\left(x_{j}-x_{i}\right)\left[f_{i}(t, x)-f_{i}\left(t, x+\left(x_{i}-x_{j}\right) e_{j}\right)\right] \\
& \geq \Psi_{i j}(t) \varepsilon_{\mathcal{K}}\left|x_{j}-x_{i}\right|
\end{aligned}
$$

with

$$
\Psi_{i j}(t)= \begin{cases}1 & \text { if } \quad t \in \psi_{(i, j)},(i, j) \in \mathcal{T}_{r} \\ 0 & \text { otherwise. }\end{cases}
$$

and

$$
\int_{\psi_{(i, j)} \cap[t, t+T]} d \tau \geq \varepsilon_{T}
$$

Remark 1 Notice that if equation (4) holds for some $T$, it holds a fortiori for all $\tilde{T}>T$.

Notice that, in the light of equation (4) this is an assumption of averaged weak connectedness across uniform time intervals, while by condition (3), the node interaction property is defined on frozen state variables across the same interval, making its verification straightforward. The following fact is well-known for the existing consensus conditions and it holds under the proposed assumption. The prove is omitted for sake of brevity and may be find elsewhere ([17]).

Lemma 1 The functions $x_{\max }(t)$ and $x_{\min }(t)$ are (respectively) monotonically non-increasing and nondecreasing.

In what follows we will present a key lemma which will allow us to later prove exponential asymptotic consensus.

Lemma 2 Let $r \in N$ be the root of the spanning tree as from Assumption 1. For all initial conditions $x(0) \in \mathbb{R}^{n}$, there exists a finite positive integer $\bar{k}$ and $\mu>0$ (uniform 
in time) such that, for all $t \geq 0$, the following holds along the solutions of (1):

$$
x_{\max }(t+\bar{k} T) \leq x_{\max }(t)-\mu\left|x_{\max }(t)-x_{r}(t)\right|
$$

and:

$$
x_{\min }(t+\bar{k} T) \geq x_{\min }(t)+\mu\left|x_{\min }(t)-x_{r}(t)\right| .
$$

Proof: We prove the Lemma for $x_{\max }(t)$, a similar argument holds for $x_{\min }(t)$. Let $\varepsilon=\varepsilon_{T} \varepsilon_{x}$, and $d(q): N \rightarrow \mathbb{N}$ denote the distance in the spanning tree of node $q$ from the root $r$ of the tree $\mathcal{T}_{r}$ as in Assumption 1. Let us deal first with nodes $q$ at distance $d(q)=1$. We consider different cases.

Case $a): x_{r}(\tau) \leq x_{q}(\tau)$, for all $\tau \in[t, t+2 T]$.

To come up with a suitable estimate we further need to consider the following subcases.

Subcase $\left.a_{1}\right) \delta_{q}(t) \leq \frac{1}{2} \delta_{r}(t)$. Define $\bar{q}(\tau) \in\{1, \ldots, n\}$ so as to fulfill $p_{\bar{q}(\tau)}(\tau)=q$. In the following expressions, the time dependence of $\bar{q}$ will be omitted for the sake of simplicity of notation. Then, for any node $q$ at distance 1 from the root it holds for all $\tau \in[t, t+2 T]$ :

$$
\begin{aligned}
& x_{q}(\tau)-x_{q}(t)=\int_{t}^{\tau} f_{q}(\theta, x(\theta)) d \theta= \\
& \int_{t}^{\tau}\left(\left[f_{q}(\theta, x(\theta))-f_{q}\left(\theta, x(\theta)+\left(x_{q}(\theta)-\right.\right.\right.\right. \\
& \left.\left.x_{p_{\bar{q}-1}(\theta)}(\theta)\right) e_{p_{\bar{q}-1}(\theta)}\right)+\left[f_{q}(\theta, x(\theta)+\right. \\
& \left.\left(x_{q}(\theta)-x_{p_{\bar{q}-1}(\theta)}(\theta)\right) e_{p_{\bar{q}-1}(\theta)}\right)-f_{q}(\theta, x(\theta)+ \\
& \left(x_{q}(\theta)-x_{p_{\bar{q}-1}(\theta)}(\theta)\right) e_{p_{\bar{q}-1}(\theta)}+\left(x_{q}(\theta)-\right. \\
& \left.\left.x_{p_{\bar{q}-2}(\theta)}(\theta)\right) e_{p_{\bar{q}-2}(\theta)}\right)+\left[f _ { q } \left(\theta, x(\theta)+\left(x_{q}(\theta)\right.\right.\right. \\
& \left.-x_{\left.p_{\bar{q}-1}(\theta)\right)}(\theta)\right) e_{p_{\bar{q}-1}(\theta)} \\
& \left.+\left(x_{q}(\theta)-x_{p_{\bar{q}-2}(\theta)}(\theta)\right) e_{p_{\bar{q}-2}(\theta)}\right)- \\
& f_{q}\left(\theta, x(\theta)+\left(x_{q}(\theta)-x_{p_{\bar{q}-1}(\theta)}(\theta)\right) e_{p_{\bar{q}-1}(\theta)}\right. \\
& +\left(x_{q}(\theta)-x_{p_{\bar{q}-2}(\theta)}(\theta)\right) e_{p_{\bar{q}-2}(\theta)} \\
& \left.+\left(x_{q}(\theta)-x_{p_{\bar{q}-3}(\theta)}(\theta)\right) e_{p_{\bar{q}-3}(\theta)}\right)+ \\
& \ldots+\left[f _ { q } \left(\theta, x(\theta)+\left(x_{q}(\theta)-x_{p_{\bar{q}-1}(\theta)}(\theta)\right) e_{p_{\bar{q}-1}(\theta)}+\ldots\right.\right. \\
& \left.+\left(x_{q}(\theta)-x_{p_{2}(\theta)}(\theta)\right) e_{p_{2}(\theta)}\right) \\
& +f_{q}\left(\theta, x(\theta)+\left(x_{q}(\theta)-x_{p_{\bar{q}-1}(\theta)}(\theta)\right) e_{p_{\bar{q}-1}(\theta)}\right. \\
& \left.\left.+\ldots+\left(x_{q}(\theta)-x_{p_{1}(\theta)}(\theta)\right) e_{p_{1}(\theta)}\right)\right] \\
& +f_{q}\left(\theta, x(\theta)+\left(x_{q}(\theta)-x_{p_{\bar{q}-1}(\theta)}(\theta)\right) e_{p_{\bar{q}-1}(\theta)}\right. \\
& \left.\left.+\ldots+\left(x_{q}(\theta)-x_{p_{1}(\theta)}(\theta)\right) e_{p_{1}(\theta)}\right)\right) d \theta \\
& +\ldots
\end{aligned}
$$

The application of Assumption 1 to each of the terms in the integrand of the previous expression (except for the last one) leads to:

$$
x_{q}(\tau)-x_{q}(t) \leq
$$

$$
\begin{aligned}
& -\int_{t}^{\tau} \sum_{j: x_{j}(\theta)<x_{q}(\theta)} \Psi_{q j}(\theta) \varepsilon_{\mathcal{K}}\left|x_{q}(\theta)-x_{j}(\theta)\right| d \theta \\
& +\int_{t}^{\tau} f_{q}\left(\theta, x(\theta)+\left(x_{q}(\theta)-x_{p_{\bar{q}-1}(\theta)}(\theta)\right) e_{p_{\bar{q}-1}(\theta)}\right. \\
& \left.+\ldots+\left(x_{q}(\theta)-x_{p_{1}(\theta)}(\theta)\right) e_{p_{1}(\theta)}\right) d \theta
\end{aligned}
$$

The former calculations are instrumental for the subsequent exploitation of uniform Lipshitz continuity of $f$ as detailed below:

$$
\begin{aligned}
& x_{q}(\tau)-x_{q}(t) \leq \\
& -\int_{t}^{\tau} \sum_{j: x_{j}(\theta)<x_{q}(\theta)} \Psi_{q j}(\theta) \varepsilon_{\mathcal{K}}\left|x_{q}(\theta)-x_{j}(\theta)\right| d \theta \\
& +\int_{t}^{\tau}\left(\left[f _ { q } \left(\theta, x(\theta)+\left(x_{q}(\theta)-x_{p_{\bar{q}-1}}\right) e_{p_{\bar{q}-1}}+\right.\right.\right. \\
& \left.\left.\ldots+\left(x_{q}(\theta)-x_{p_{1}(\theta)}(\theta)\right) e_{p_{1}(\theta)}\right)-f_{q}\left(\theta, x_{q}(\theta) \mathbf{1}\right)\right] \\
& \left.+f_{q}\left(\theta, x_{q}(\theta) \mathbf{1}\right)\right) d \theta .
\end{aligned}
$$

Being $f_{q}\left(\theta, x_{q}(\theta) \mathbf{1}\right)=0$, it results:

$$
\begin{aligned}
& x_{q}(\tau)-x_{q}(t) \leq \\
& -\int_{t}^{\tau} \sum_{j: x_{j}(\theta)<x_{q}(\theta)} \Psi_{q j}(\theta) \varepsilon_{\mathcal{K}}\left|x_{q}(\theta)-x_{j}(\theta)\right| d \theta \\
& -L \int_{t}^{\tau} \sum_{j: x_{j}(\theta) \geq x_{q}(\theta)}\left[x_{q}(\theta)-x_{j}(\theta)\right] d \theta \\
& \leq-\int_{t}^{\tau} \sum_{j: x_{j}(\theta)<x_{q}(\theta)} \Psi_{q j}(\theta) \varepsilon_{\mathcal{K}}\left|x_{q}(\theta)-x_{j}(\theta)\right| d \theta \\
& -L \int_{t}^{\tau} \sum_{j: x_{j}(\theta) \geq x_{q}(\theta)}\left[x_{q}(\theta)-x_{\max }(t)\right] d \theta \\
& \leq-\int_{t}^{\tau} \sum_{j: x_{j}(\theta)<x_{q}(\theta)} \Psi_{q j}(\theta) \varepsilon_{\mathcal{K}}\left|x_{q}(\theta)-x_{j}(\theta)\right| d \theta \\
& -(n-1) L \int_{t}^{\tau}\left[x_{q}(\theta)-x_{\max }(t)\right] d \theta,
\end{aligned}
$$

with $L$ denotes the (time-independent) Lipschitz constant of $f_{q}$. In particular for all $\tau \in[t+T, t+2 T]$ we see that:

$$
\begin{aligned}
& x_{q}(\tau)-x_{\max }(t) \leq x_{q}(\tau)-x_{q}(t) \\
& \leq-\int_{t}^{\tau} \sum_{j: x_{j}(\theta)<x_{q}(\theta)} \Psi_{q j}(\theta) \varepsilon_{\mathcal{K}}\left|x_{q}(\theta)-x_{j}(\theta)\right| d \theta \\
& -(n-1) L \int_{t}^{\tau}\left[x_{q}(\theta)-x_{\max }(t)\right] d \theta \\
& \leq-\int_{t}^{\tau} \Psi_{q r}(\theta) \varepsilon_{\mathcal{K}}\left|x_{q}(\theta)-x_{r}(\theta)\right| d \theta \\
& -(n-1) L \int_{t}^{\tau}\left[x_{q}(\theta)-x_{\max }(t)\right] d \theta .
\end{aligned}
$$


By the triangular inequality it holds:

$-\left|x_{q}(\theta)-x_{r}(\theta)\right| \leq-\left|x_{\max }(t)-x_{r}(\theta)\right|+\left|x_{\max }(t)-x_{q}(\theta)\right|$, moreover, by Lipschitz continuity of $f_{r}$, we may infer:

$$
\left|x_{\max }(t)-x_{r}(\theta)\right| \geq e^{-L(\theta-t)}\left|x_{\max }(t)-x_{r}(t)\right| .
$$

Combining the above inequalities, we may restate the bound for $x_{q}(\tau)-x_{\max }(t)$ expressed in (7) as detailed below:

$$
\begin{aligned}
& x_{q}(\tau)-x_{\max }(t) \leq \\
& -\int_{t}^{\tau} \Psi_{q r}(\theta) \varepsilon_{\mathcal{K}} e^{-L(\theta-t)}\left|x_{\max }(t)-x_{r}(t)\right| d \theta \\
& -\int_{t}^{\tau} \Psi_{q r}(\theta) \varepsilon_{\mathcal{K}}\left[x_{q}(\theta)-x_{\max }(t)\right] d \theta \\
& -(n-1) L \int_{t}^{\tau}\left[x_{q}(\theta)-x_{\max }(\theta)\right] d \theta \\
& \leq-\varepsilon_{\mathcal{K}} e^{-2 L T}\left|x_{\max }(t)-x_{r}(t)\right| \int_{t}^{\tau} \Psi_{q r}(\theta) d \theta \\
& -\varepsilon_{\mathcal{K}} \int_{t}^{\tau}\left[x_{q}(\theta)-x_{\max }(t)\right] d \theta \\
& -(n-1) L \int_{t}^{\tau}\left[x_{q}(\theta)-x_{\max }(t)\right] d \theta \\
& \leq-\varepsilon e^{-2 L T}\left|x_{\max }(t)-x_{r}(t)\right| \\
& -\varepsilon_{\mathcal{K}} \int_{t}^{\tau}\left[x_{q}(\theta)-x_{\max }(t)\right] d \theta \\
& -(n-1) L \int_{t}^{\tau}\left[x_{q}(\theta)-x_{\max }(t)\right] d \theta
\end{aligned}
$$

with $\varepsilon=\varepsilon_{\mathcal{K}} \varepsilon_{T}$. By defining $\Delta(\tau)=\int_{t}^{\tau}\left[x_{q}(\theta)-\right.$ $\left.x_{\max }(t)\right] d \theta$ we can recast equation (8) as:

$$
\begin{aligned}
& \frac{d}{d \tau} \Delta(\tau) \leq-\varepsilon e^{-2 L T}\left|x_{\max }(t)-x_{r}(t)\right| \\
& -\left((n-1) L+\varepsilon_{\mathcal{K}}\right) \Delta(\tau),
\end{aligned}
$$

which holds for all $\tau \in[t+T, t+2 T]$. Since $\Delta(t+T) \leq$ 0 , by a standard comparison principle we see that:

$$
\Delta(\tau) \leq-\mu_{\Delta}(\tau)\left|x_{\max }(t)-x_{r}(t)\right|,
$$

with

$$
\mu_{\Delta}(\tau)=e^{-2 L T} \frac{\varepsilon\left[1-e^{-\left((n-1) L+\varepsilon_{\mathcal{K}}\right)(\tau-T-t)}\right]}{\left((n-1) L+\varepsilon_{\mathcal{K}}\right)},
$$

which holds for all $\tau \in[t+T, t+2 T]$. In particular, for $\tau=t+2 T$ equation (9) yields:

$$
\Delta(t+2 T) \leq-\mu_{\Delta}\left|x_{\max }(t)-x_{r}(t)\right|,
$$

with

$$
\mu_{\Delta}=\frac{\varepsilon}{\left((n-1) L+\varepsilon_{\mathcal{K}}\right)} e^{-2 L T}\left[1-e^{-\left((n-1) L+\varepsilon_{\mathcal{K}}\right) T}\right]
$$

From the mean value theorem it results:

$$
\exists t^{*} \in[t, t+2 T]: x_{q}\left(t^{*}\right)-x_{\max }(t)=\frac{\Delta(t+2 T)}{2 T} .
$$

By Lipschitz continuity of $f$, convergence of $x_{q}(t)$ towards $x_{\max }(t)$ is at most exponential in time and therefore we may infer:

$$
x_{q}(t+2 T)-x_{\max }(t) \leq\left(x_{q}\left(t^{*}\right)-x_{\max }(t)\right) e^{-2 L T} .
$$

From (11) and (12) it results:

$$
x_{q}(t+2 T)-x_{\max }(t) \leq \frac{\Delta(t+2 T)}{2 T} e^{-2 L T}
$$

Finally, in order to derive an estimate of how decreasing is $x_{q}(t)$ which is uniform in time we combine (13) and (10) and obtain:

$$
x_{q}(t+2 T)-x_{\max }(t) \leq-\mu_{a_{1}} \delta_{r}(t),
$$

with $\mu_{a_{1}}=e^{-4 L T \frac{\varepsilon\left[1-e^{-\left((n-1) L+\varepsilon_{\mathcal{K}}\right) T}\right]}{2\left((n-1) L+\varepsilon_{\mathcal{K}}\right) T}}$ and $\delta_{r}(t)=$ $\left|x_{\max }(t)-x_{r}(t)\right|$.

Subcase $\left.a_{2}\right) \delta_{q}(t)>\frac{1}{2} \delta_{r}(t)$

In this scenario, by Lipschitz continuity of $f$, convergence of $x_{q}(\tau)$ towards the value $x_{\max }(t)$ is at most exponential, and therefore we may infer:

$$
\left|x_{\max }(t)-x_{q}(t+2 T)\right| \geq e^{-2 L T}\left|x_{\max }(t)-x_{q}(t)\right| .
$$

that yields:

$$
\begin{aligned}
& x_{q}(t+2 T) \leq x_{\max }(t)-e^{-2 L T}\left|x_{\max }(t)-x_{q}(t)\right| \\
& =x_{\max }(t)-e^{-2 L T} \delta_{q}(t) \\
& \leq x_{\max }(t)-\mu_{a_{2}} \delta_{r}(t) .
\end{aligned}
$$

with $\mu_{a_{2}}=\frac{1}{2} e^{-2 L T}$ and $\delta_{r}(t)=\left|x_{\max }(t)-x_{r}(t)\right|$.

Case $b): x_{r}(\tau) \geq x_{q}(\tau)$ for all $\tau \in[t, t+2 T]$

In this case considering that $\delta_{q}(t) \geq \delta_{r}(t)$ and exploiting Lipschitz continuity of $f$, we may infer:

$$
\begin{aligned}
& x_{q}(t+2 T) \leq x_{\max }(t)-e^{-2 L T}\left|x_{\max }(t)-x_{q}(t)\right| \\
& =x_{\max }(t)-e^{-2 L T} \delta_{q}(t) \leq x_{\max }(t)-\mu_{b} \delta_{r}(t),
\end{aligned}
$$

with $\mu_{b}=e^{-2 L T}$.

Case $c): \exists \bar{\tau} \in(0,2 T]$ such that $x_{q}(t+\bar{\tau})=x_{r}(t+\bar{\tau})$. By Lipschitz continuity of $f$, convergence of $x_{r}$ and $x_{q}$ towards the value $x_{\max }(t)$ is at most exponential. This, along with assumption $x_{q}(t+\bar{\tau})=x_{r}(t+\bar{\tau})$, yields:

$$
\begin{aligned}
& \left|x_{\max }(t)-x_{q}(t+2 T)\right| \geq \\
& e^{-L(2 T-\bar{\tau})}\left|x_{\max }(t)-x_{q}(t+\bar{\tau})\right| \\
& =e^{-L(2 T-\bar{\tau})}\left|x_{\max }(t)-x_{r}(t+\bar{\tau})\right| \\
& \geq e^{-2 L T}\left|x_{\max }(t)-x_{r}(t)\right|,
\end{aligned}
$$

and therefore $x_{q}(t+2 T) \leq x_{\max }(t)-\mu_{c} \delta_{r}(t)$ with $\mu_{c}=$ $e^{-2 L T}$.

Therefore, in any of cases $a, b$ and $c$ it results:

$$
x_{q}(t+2 T) \leq x_{\max }(t)-\mu_{1} \delta_{r}(t)
$$


or in other terms:

$$
\left|x_{\max }(t)-x_{q}(t+2 T)\right| \geq \mu_{1} \delta_{r}(t)
$$

with $\mu_{1}=\min \left\{\mu_{a_{1}}, \mu_{a_{2}}, \mu_{b}, \mu_{c}\right\}$ and $\delta_{r}(t)=$ $\left|x_{\max }(t)-x_{r}(t)\right|$. Next we deal with nodes $k \in N$ with $d(k)=2$. Let $q$ be such that $d(q)=1$ and $(q, k) \in \mathcal{T}_{r}$. We consider different cases.

Case $a): x_{k}(t+\tau) \geq x_{q}(t+\tau)$, for all $\tau \in[2 T, 4 T]$ Subcase $\left.a_{1}\right): \delta_{k}(t+2 T) \leq \frac{1}{2} \delta_{q}(t+2 T)$. For node $k$, let $\bar{k}(\tau)$ be such that $p_{\bar{k}(\tau)}(\tau)=k$. In the following we omit the time dependence of $\bar{k}$ for the sake of simplicity of notation. Then, the following holds for all $\tau \in[t+2 T, t+4 T]:$

$$
\begin{aligned}
& x_{k}(\tau)-x_{k}(t+2 T)=\int_{t+2 T}^{\tau} f_{k}(\theta, x(\theta)) d \theta \\
& =\int_{t+2 T}^{\tau}\left(\left[f_{k}(\theta, x(\theta))-f_{k}(\theta, x(\theta)\right.\right. \\
& \left.\left.+\left(x_{k}(\theta)-x_{p_{\bar{k}-1}(\theta)}(\theta)\right) e_{p_{\bar{k}-1}(\theta)}\right)\right] \\
& +\left[f_{k}\left(\theta, x(\theta)+\left(x_{k}(\theta)-x_{p_{\bar{k}-1}(\theta)}(\theta)\right) e_{p_{\bar{k}-1}(\theta)}\right)\right. \\
& -f_{k}\left(\theta, x(\theta)+\left(x_{k}(\theta)-x_{p_{\bar{k}-1}(\theta)}(\theta)\right) e_{p_{\bar{k}-1}(\theta)}\right. \\
& \left.\left.+\left(x_{k}(\theta)-x_{p_{\bar{k}-2}(\theta)}(\theta)\right) e_{p_{\bar{k}-2}(\theta)}\right)\right] \\
& +\left[f _ { k } \left(\theta, x(\theta)+\left(x_{k}(\theta)-x_{p_{\bar{k}-1}(\theta)}(\theta)\right) e_{p_{\bar{k}-1}(\theta)}\right.\right. \\
& \left.+\left(x_{k}(\theta)-x_{p_{\bar{k}-2}(\theta)}(\theta)\right) e_{p_{\bar{k}-2}(\theta)}\right) \\
& -f_{k}\left(\theta, x(\theta)+\left(x_{k}(\theta)-x_{p_{\bar{k}-1}(\theta)}(\theta)\right) e_{p_{\bar{k}-1}(\theta)}+\right. \\
& \left(x_{k}(\theta)-x_{p_{\bar{k}-2}(\theta)}(\theta)\right) e_{p_{\bar{k}-2}(\theta)} \\
& \left.\left.+\left(x_{k}(\theta)-x_{p_{\bar{k}-3}(\theta)}(\theta)\right) e_{p_{\bar{k}-3}(\theta)}\right)\right] \\
& +\ldots+\left[f _ { k } \left(\theta, x(\theta)+\left(x_{k}(\theta)-x_{p_{\bar{k}-1}(\theta)}(\theta)\right) e_{p_{\bar{k}-1}(\theta)}+\right.\right. \\
& \left.\ldots+\left(x_{k}(\theta)-x_{p_{2}(\theta)}(\theta)\right) e_{p_{2}(\theta)}\right) \\
& -f_{k}\left(\theta, x(\theta)+\left(x_{k}(\theta)-x_{p_{\bar{k}-1}(\theta)}(\theta)\right) e_{p_{\bar{k}-1}(\theta)}+\right. \\
& \left.\left.\ldots+\left(x_{k}(\theta)-x_{p_{1}(\theta)}(\theta)\right) e_{p_{1}(\theta)}\right)\right] \\
& +f_{k}\left(\theta, x(\theta)+\left(x_{k}(\theta)-x_{p_{\bar{k}-1}(\theta)}(\theta)\right) e_{p_{\bar{k}-1}(\theta)}+\right. \\
& \left.\left.\ldots+\left(x_{k}(\theta)-x_{p_{1}(\theta)}(\theta)\right) e_{p_{1}(\theta)}\right)\right) d \theta \\
& \leq-\int_{t+2 T}^{\tau} \sum_{j: x_{j}(\theta)<x_{k}(\theta)} \Psi_{k j}(\theta) \varepsilon_{\mathcal{K}}\left|x_{k}(\theta)-x_{j}(\theta)\right| d \theta \\
& +\int_{t+2 T}^{\tau} f_{k}\left(\theta, x(\theta)+\left(x_{k}(\theta)-x_{p_{\bar{k}-1}(\theta)}(\theta)\right) e_{p_{\bar{k}-1}(\theta)}\right. \\
& \left.+\ldots+\left(x_{k}(\theta)-x_{p_{1}(\theta)}(\theta)\right) e_{p_{1}(\theta)}\right) d \theta \\
& \leq-\int_{t+2 T}^{\tau} \sum_{j: x_{j}(\theta)<x_{k}(\theta)} \Psi_{k j}(\theta) \varepsilon_{\mathcal{K}}\left|x_{k}(\theta)-x_{j}(\theta)\right| d \theta+ \\
& \int_{t+2 T}^{\tau}\left(\left[f _ { k } \left(\theta, x(\theta)+\left(x_{k}-x_{p_{\bar{k}-1}(\theta)}(\theta)\right) e_{p_{\bar{k}-1}(\theta)}+\right.\right.\right. \\
& \left.\left.\ldots+\left(x_{k}(\theta)-x_{p_{1}(\theta)}(\theta)\right) e_{p_{1}(\theta)}\right)-f_{k}\left(\theta, x_{k}(\theta) \mathbf{1}\right)\right] \\
& +f_{k}\left(\theta, x_{k}(\theta) \mathbf{1}\right) d \theta
\end{aligned}
$$

Being $f_{k}\left(\theta, x_{k}(\theta) \mathbf{1}\right)=0$, it results:

$$
\begin{aligned}
& x_{k}(\tau)-x_{k}(t) \\
& \leq-\int_{t+2 T}^{\tau} \sum_{j: x_{j}(\theta)<x_{k}(\theta)} \Psi_{k j}(\theta) \varepsilon_{\mathcal{K}}\left|x_{k}(\theta)-x_{j}(\theta)\right| d \theta \\
& -L \int_{t+2 T}^{\tau} \sum_{j: x_{j}(\theta) \geq x_{k}(\theta)}\left[x_{k}(\theta)-x_{j}(\theta)\right] d \theta \\
& \leq-\int_{t+2 T}^{\tau} \sum_{j: x_{j}(\theta)<x_{k}(\theta)} \Psi_{k j}(\theta) \varepsilon_{\mathcal{K}}\left|x_{k}(\theta)-x_{j}(\theta)\right| d \theta \\
& -L \int_{t+2 T}^{\tau} \sum_{j: x_{j}(\theta) \geq x_{k}(\theta)}\left[x_{k}(\theta)-x_{\max }(t)\right] d \theta \\
& \leq-\int_{t+2 T}^{\tau} \sum_{j: x_{j}(\theta)<x_{k}(\theta)} \Psi_{k j}(\theta) \varepsilon_{\mathcal{K}}\left|x_{k}(\theta)-x_{j}(\theta)\right| d \theta \\
& -(n-1) L \int_{t+2 T}^{\tau}\left[x_{k}(\theta)-x_{\max }(t)\right] d \theta
\end{aligned}
$$

where again $L$ denotes the (time-independent) Lipschitz constant of $f_{k}$.

In particular, exploiting:

$$
x_{k}(t+2 T) \leq x_{\max }(t+2 T) \leq x_{\max }(t),
$$

and resuming the series of inequalities in (16), we see that for all $\tau \in[t+3 T, t+4 T]$ it holds:

$$
\begin{aligned}
& x_{k}(\tau)-x_{\max }(t) \leq x_{k}(\tau)-x_{k}(t+2 T) \\
& \leq-\int_{t+2 T}^{\tau} \sum_{j: x_{j}(\theta)<x_{k}(\theta)} \Psi_{k j}(\theta) \varepsilon_{\mathcal{K}}\left|x_{k}(\theta)-x_{j}(\theta)\right| d \theta \\
& -(n-1) L \int_{t+2 T}^{\tau}\left[x_{k}(\theta)-x_{\max }(t)\right] d \theta \\
& \leq-\int_{t+2 T}^{\tau} \Psi_{k q}(\theta) \varepsilon_{\mathcal{K}}\left|x_{k}(\theta)-x_{q}(\theta)\right| d \theta \\
& -(n-1) L \int_{t+2 T}^{\tau}\left[x_{k}(\theta)-x_{\max }(t)\right] d \theta
\end{aligned}
$$

By the triangular inequality:

$-\left|x_{k}(\theta)-x_{q}(\theta)\right| \leq-\left|x_{\max }(t)-x_{q}(\theta)\right|+\left|x_{\max }(t)-x_{k}(\theta)\right|$, moreover, by Lipschitz continuity of $f$, we may infer: $\left|x_{\max }(t)-x_{q}(\theta)\right| \geq e^{-L(\theta-(t+2 T)}\left|x_{\max }(t)-x_{q}(t+2 T)\right|$. Therefore, substituting the previous inequalities in (17), we have:

$$
\begin{aligned}
& x_{k}(\tau)-x_{\max }(t) \leq \\
& -\int_{t+2 T}^{\tau} \Psi_{k q}(\theta) \varepsilon_{\mathcal{K}} e^{-L(\theta-t)}\left|x_{\max }(t)-x_{q}(t+2 T)\right| d \theta \\
& -\int_{t+2 T}^{\tau} \Psi_{k q}(\theta) \varepsilon_{\mathcal{K}}\left[x_{k}(\theta)-x_{\max }(t)\right] d \theta \\
& -(n-1) L \int_{t+2 T}^{\tau}\left[x_{k}(\theta)-x_{\max }(\theta)\right] d \theta
\end{aligned}
$$




$$
\begin{aligned}
& \leq-\varepsilon_{\mathcal{K}} e^{-2 L T}\left|x_{\max }(t)-x_{q}(t+2 T)\right| \int_{t+2 T}^{\tau} \Psi_{k q}(\theta) d \theta \\
& -\varepsilon_{\mathcal{K}} \int_{t+2 T}^{\tau}\left[x_{k}(\theta)-x_{\max }(t)\right] d \theta \\
& -(n-1) L \int_{t+2 T}^{\tau}\left[x_{k}(\theta)-x_{\max }(t)\right] d \theta \\
& \leq-\varepsilon e^{-2 L T}\left|x_{\max }(t)-x_{q}(t+2 T)\right| \\
& -\varepsilon_{\mathcal{K}} \int_{t+2 T}^{\tau}\left[x_{k}(\theta)-x_{\max }(t)\right] d \theta \\
& -(n-1) L \int_{t+2 T}^{\tau}\left[x_{k}(\theta)-x_{\max }(t)\right] d \theta
\end{aligned}
$$

By defining $\Delta(\tau)=\int_{t+2 T}^{\tau}\left[x_{k}(\theta)-x_{\max }(t)\right] d \theta$ we can recast the above equation as:

$$
\begin{aligned}
\frac{d}{d \tau} \Delta(\tau) & \leq-\left((n-1) L+\varepsilon_{x}\right) \Delta(\tau) \\
& -\varepsilon e^{-2 L T}\left|x_{\max }(t)-x_{q}(t+2 T)\right|,
\end{aligned}
$$

which holds for all $\tau \in[t+3 T, t+4 T]$. Since $\Delta(t+$ $3 T) \leq 0$, by a standard comparison principle we see that:

$$
\Delta(\tau) \leq-\mu_{\Delta}(\tau)\left|x_{\max }(t)-x_{q}(t+2 T)\right|,
$$

with

$$
\mu_{\Delta}(\tau)=e^{-2 L T} \frac{\varepsilon\left[1-e^{-\left((n-1) L+\varepsilon_{\mathcal{K}}\right)(\tau-T-t)}\right]}{\left((n-1) L+\varepsilon_{\mathcal{K}}\right)},
$$

which holds for all $\tau \in[t+3 T, t+4 T]$. In particular, for $\tau=t+4 T$ equation (19) yields:

$$
\Delta(t+4 T) \leq-\mu_{\Delta}\left|x_{\max }(t)-x_{q}(t+2 T)\right|,
$$

with

$$
\mu_{\Delta}=\frac{\varepsilon}{\left((n-1) L+\varepsilon_{\mathcal{K}}\right)} e^{-2 L T}\left[1-e^{-\left((n-1) L+\varepsilon_{\mathcal{K}}\right) T}\right] .
$$

By applying the mean value theorem and exploiting Lipschitz continuity, it results:

$$
\begin{aligned}
& x_{k}(t+4 T)-x_{\max }(t) \leq \frac{\Delta(t+4 T)}{2 T} e^{-2 L T} \\
& \leq-\mu_{a_{1}}\left|x_{\max }(t)-x_{q}(t+2 T)\right|
\end{aligned}
$$

with $\mu_{a_{1}}=e^{-4 L T \frac{\varepsilon\left[1-e^{-\left((n-1) L+\varepsilon_{\mathcal{K}}\right) T}\right]}{2\left((n-1) L+\varepsilon_{\mathcal{K}}\right) T}}$.

Subcase $\left.a_{2}\right): \delta_{k}(t+2 T) \geq \frac{1}{2} \delta_{q}(t+2 T)$. In this scenario taking into account that by Lipschitz continuity of $f$ it results $\left|x_{\max }(t+4 T)-x_{k}(t+4 T)\right| \geq$ $e^{-2 L T}\left|x_{\max }(t+2 T)-x_{k}(t+2 T)\right|$, we may infer:

$$
\begin{aligned}
& x_{k}(t+4 T)-x_{\max }(t) \\
& =x_{k}(t+4 T)-x_{\max }(t+2 T) \\
& -\left[x_{\max }(t)-x_{\max }(t+2 T)\right] \\
& \leq x_{k}(t+4 T)-x_{\max }(t+4 T) \\
& -\left[x_{\max }(t)-x_{\max }(t+2 T)\right]
\end{aligned}
$$

$$
\begin{aligned}
& \leq-e^{-2 L T}\left|x_{\max }(t+2 T)-x_{k}(t+2 T)\right| \\
& -\left[x_{\max }(t)-x_{\max }(t+2 T)\right] \\
& =-e^{-2 L T} \delta_{k}(t+2 T)-\left[x_{\max }(t)-x_{\max }(t+2 T)\right] \\
& \leq-e^{-2 L T} \frac{1}{2} \delta_{q}(t+2 T) \\
& -\left[x_{\max }(t)-x_{\max }(t+2 T)\right] \\
& =-e^{-2 L T} \frac{1}{2}\left[x_{\max }(t+2 T)-x_{q}(t+2 T)\right] \\
& -\left[x_{\max }(t)-x_{\max }(t+2 T)\right] \\
& \leq-e^{-2 L T} \frac{1}{2}\left[x_{\max }(t)-x_{q}(t+2 T)\right] \\
& +e^{-2 L T} \frac{1}{2}\left[x_{\max }(t)-x_{\max }(t+2 T)\right] \\
& -\left[x_{\max }(t)-x_{\max }(t+2 T)\right] \\
& =-\mu_{a_{2}}\left[x_{\max }(t)-x_{q}(t+2 T)\right] \\
& -\left(1-\mu_{a_{2}}\right)\left[x_{\max }(t)-x_{\max }(t+2 T)\right] \\
& \leq-\mu_{a_{2}}\left[x_{\max }(t)-x_{q}(t+2 T)\right]
\end{aligned}
$$

being $\mu_{a_{2}}=e^{-2 L T} \frac{1}{2}<1$.

Case $b): x_{q}(t+\tau) \geq x_{k}(t+\tau) \tau \in[2 T, 4 T]$

In this case, we may infer:

$x_{k}(t+4 T) \leq x_{\max }(t)-e^{-2 L T}\left|x_{\max }(t)-x_{k}(t+2 T)\right|$ $\leq x_{\max }(t)-\mu_{b}\left|x_{\max }(t)-x_{q}(t+2 T)\right|$,

with $\mu_{b}=e^{-2 L T}$.

Case $c): x_{q}(t+\bar{\tau})=x_{k}(t+\bar{\tau})$ for some $\bar{\tau} \in(2 T, 4 T]$. By Lipschitz continuity of $f$, it results:

$$
\begin{aligned}
& \left|x_{\max }(t)-x_{k}(t+4 T)\right| \geq \\
& e^{-L(4 T-\bar{\tau})}\left|x_{\max }(t)-x_{k}(t+\bar{\tau})\right| \\
& e^{-L(4 T-\bar{\tau})}\left|x_{\max }(t)-x_{q}(t+\bar{\tau})\right| \\
& \geq e^{-2 L T}\left|x_{\max }(t)-x_{q}(t+2 T)\right| \\
& =\mu_{c}\left|x_{\max }(t)-x_{q}(t+2 T)\right| .
\end{aligned}
$$

with $\mu_{c}=e^{-2 L T}$.

Therefore, in any of cases $a, b$ and $c$ it results:

$x_{k}(t+4 T) \leq x_{\max }(t)-\mu_{2}\left|x_{\max }(t)-x_{q}(t+2 T)\right|$

with $\mu_{2}=\min \left\{\mu_{a_{1}}, \mu_{a_{2}}, \mu_{b}, \mu_{c}\right\}$.

Consequently, in order to derive an estimate of how decreasing is $x_{k}(t)$ which is uniform in time by combining (24) and (15) we obtain:

$$
x_{k}(t+4 T)-x_{\max }(t) \leq-\mu_{1} \mu_{2}\left|x_{\max }(t)-x_{r}(t)\right| .
$$

A similar procedure can be used to construct an estimate of the convergence rate for an arbitrary node at distance $d(k)+1$ based on the estimate for nodes at distance $d(k)$. By induction, for any node $k$ at distance $d(k)$ from the 
root, the following inequality holds:

$$
\begin{aligned}
& x_{k}(t+2 d(k) T)-x_{\max }(t) \\
& \leq-\left(\prod_{i=1}^{d(k)} \mu_{i}\right)\left|x_{\max }(t)-x_{r}(t)\right| \\
& =-\mu(d(k))\left|x_{\max }(t)-x_{r}(t)\right|,
\end{aligned}
$$

with $\mu(d(k))=\prod_{i=1}^{d(k)} \mu_{i}$ being a positive constant for any fixed $d(k)$.

Given the fact that only a finite number of agents are present and by Assumption 1 every agent $k$ has a finite distance from the root, a uniform estimate of the convergence rate can be provided.

Estimate (26) is still not of the form needed to prove our claim as the estimated rate of contraction is $d(k)$ dependent and the number of $T$ intervals needed in order to guarantee such decrease in $x_{\max }$ is proportional to $d(k)$.

Nevertheless, $\mu(d(k)) \in(0,1)$ for any $d(k)$ and by monotonicity of $x_{\max }(t)$ (see Lemma 1) and finiteness of the number of agents, one can take without loss of generality $\bar{k}:=2(n-1)$ and $\mu=\mu(\bar{k} / 2)=\mu(n-1)$. This concludes the proof of the Lemma.

Theorem 1 Consider the network modeled by equations (1), if Assumptions 1 hold, then the equilibrium set is uniformly exponentially stable and, for any initial condition $x(0), x(t)$ converges to an agreement equilibrium state with the following contraction rate:

$$
V(x(t+s \bar{k} T)) \leq(1-\hat{\mu})^{s} V(x(t)) .
$$

with $\hat{\mu}=\mu(n-1)$.

The result is proven by using Lemma 1, Lemma 2 and Lyapunov argument. The proof is omitted for sake of brevity and may be find elsewhere ([17]). An extended version of the paper including the omitted profs and numerical examples and discussions has been submitted ([17]).

\section{CONCLUSIONS}

In this paper, we introduced a criterion for asymptotic exponential consensus avoiding the assumption of cooperativity. This is widely adopted in the literature, either explicitly or implicitly, and implies monotonicity of the system's flow with respect to initial conditions. The criterion extends to this class of nonlinear scenario the possibility of guaranteeing consensus through an averaged notion of connectivity.

\section{REFERENCES}

[1] R.M. Murray, Recent Research in Cooperative Control of Multivehicle Systems, Journal of Dynamic Systems, Measurement, and Control, 129, 5, 2007

[2] W. Ren, R.W. Beard, and E. M. Atkins, Information consensus in multivehicle control, IEEE Control Syst. Mag., 27, 2, 2007.
[3] Sarlette, A.; Bonnabel, S.; Sepulchre, R. Coordinated Motion Design on Lie Groups, IEEE Transactions on Automatic Control, $55,5,2010$

[4] Sarlette, A.; Bonnabel, S.; Sepulchre, R. Degree Fluctuations and the Convergence Time of Consensus Algorithms, IEEE Transactions on Automatic Control, 58, 10, 2013

[5] Saber R.O., Murray R.M. Consensus Problems in Networks of Agents with Switching Topology and Time-Delays, IEEE Transactions on Automatic Control, 49, 92004.

[6] Jadbabaie, A., Lin, J. and Morse A.S. Coordination of groups of mobile agents using nearest neighbor rules. IEEE Transactions on Automatic Control, 48, 6, 2003

[7] F.H. Clarke, Y.S. Ledyaev, R.J. Stern and P.R. Wolenski, Nonsmooth Analysis and Control Theory, Springer, 1998.

[8] F. Blanchini and S. Miani, Set Theoretic Methods in Control, Systems and Control Foundations and Applications, Birkhauser, 2008.

[9] Scardovi, L., Sepulchre, R. Synchronization in networks of identical linear systems Automatica, 45, 11, 2009

[10] Li, Z., and Chen, G. Global synchronization and asymptotic stability of complex dynamical networks, IEEE Transactions on Circuits and Systems II, 53, 1, 2006

[11] Chopra, N., Spong, M. Passivity Based Control of Multi-Agent Systems. Advances in Robot Control From Everyday Physics in Human-Like Movements, 107-134, 2007.

[12] L. Moreau. Stability of multiagent systems with time-dependent communication links. IEEE Transactions on Automatic Control, 50, 2, 2005.

[13] V. D. Blondel, J. M. Hendrickx, A. Olshevsky, and J. N. Tsitsiklis. Convergence in multiagent coordination, consensus, and focking. In IEEE Conference Decision and Control and European Control Conference, 2005.

[14] D. Angeli, P.A. Bliman, Tight estimates for convergence of some non-stationary consensus algorithms, Systems \& Control Letters, $57,12,2008$

[15] L. Moreau. Stability of continuous-time distributed consensus algorithms, Proc. 43rd IEEE Conf. Decision and Control, 2004.

[16] S. Manfredi and D. Angeli. Frozen State Conditions for Asymptotic Consensus of Time-Varying Cooperative Nonlinear Networks. 52nd IEEE Conference on Decision and Control, pp. 1325 - 1330, ISSN:0743-1546, DOI:10.1109/CDC.2013.6760066, December 10-13, 2013, Firenze, Italy

[17] S. Manfredi and D. Angeli. A Criterion foe exponential consensus of time-varying non-cooperative nonlinear networks. submitted to Journal, 2015

[18] J. M. Hendrickx and J. N. Tsitsiklis. Convergence of typesymmetric and cut-balanced consensus seeking systems. IEEE Transactions on Automatic Control, 58, 1, 2013

[19] Z. Qu, J. Chunyu, and J. Wang. Nonlinear cooperative control for consensus of nonlinear and heterogeneous systems. In Proceedings of the 46th IEEE Conference on Decision and Control, pages 2301-2308, New Orleans, USA, 2007.

[20] U. Munz, A. Papachristodoulou, and F. Allgower, Robust Consensus Controller Design for Nonlinear Relative Degree Two Multi-Agent Systems With Communication Constraints, IEEE Transactions on Automatic Control, 56, 1, 2011.

[21] Y. Cao and W. Ren, Distributed Multi-Agent Coordination: A Comparison Lemma Based Approach, in Proceedings of the IEEE American Control Conference, San Francisco, CA, June 2011.

[22] J.-J. E. Slotine and W. Wang, A study of synchronization and group cooperation using partial contraction theory, in Cooperative Control (V. Kumar, N. E. Leonard, and A. S. Morse, eds.), vol. 309, Springer-Verlag Series: Lecture Notes in Control and Information Sciences, 2004.

[23] C. Altafini, Consensus problems on networks with antagonistic interactions, IEEE Transactions on Automatic Control, Vol. 58, No. 4, 2013.

[24] H. L. Smith. Monotone Dynamical Systems: An Introduction to the Theory of Competitive and Cooperative Systems, Volume 41 of Mathematical Surveys and Monographs. AMS, Providence, RI, 1995. 